\title{
The muscles' relaxation characteristics improvement of belt wrestlers using exercises on the basis of the instantaneous simulators
}

\author{
Radik Valinurov, Yuriy Denisenko*, Aydar Akhmetov, Irek Gilmutdinov, Natalya Seliverstova \\ Naberezhnye Chelny State Pedagogical University, 423806, Naberezhnye Chelny, Russia
}

\begin{abstract}
In modern times, qualified athletes reached high level of physical fitness. That's why, the greater increase in the volume and intensity of the training process becomes difficult task. The first step is to develop the search for new forms of the training process organization. They create optimal conditions for the full-value realization of the adaptive possibilities and the restoration of energy resources. Therefore, it is very important to search for fundamentally new means and methods to improve the physical performance of athletes. One of them is often power simulators, in which the resistance force is formed by a set of brakes that have significant inertia. However, special research and experience in sports practice indicate that the use of traditional strength training equipment, although it contributes to the development of strength quality. At the same time negatively affects the development of speed and endurance. The problem of finding new tools that cannot slow down the development of speed and endurance during the development of strength qualities remains urgent. Among the few tools of this kind, special attention should be paid to exercises that help improve the relaxation characteristics of the muscles. A number of studies showed the beneficial effect of special exercises that improve the relaxation characteristics (relaxation rate) of skeletal muscles, and contribute to improving the effectiveness of special physical performance and improving sports results.
\end{abstract}

\section{Introduction}

Wrestling is characterized by speed-power efforts of variable power over a short period of time. There are large muscle strains associated with breath delays when pressing, as well as static forces both in individual positions (wrestling bridge), and in the process of overcoming resistance during wrestling. The current work develops the most effective load planning to increase speed and strength, as well as the structure of funds. That is optimal for the development of these qualities [1-3].

According to experts, in wrestling, the leading physical quality is the speed and strength qualities of the athlete. Their development serves as the basis for the successful implementation of technical and tactical actions in competitive events. The wrestler needs specific manifestations of speed and strength qualities. It is also necessary to develop mobility, speed, coordination, and stability in parallel. This is possible through strength exercises in specific poses of wrestling technique, in a wrestling situation [2-5].

The use of traditional means and methods for the development of physical qualities does not always allow to combine the work of muscles in the conditions of physical qualities combined development with the improvement of the competitive exercise technique. At the same time, when using training devices and technical means of urgent information, it is possible to achieve a very large effect in simultaneously increasing the level of technical and physical fitness of athletes. The training devices using in the practice of sports confirmed their effectiveness in improving the physical qualities of athletes. They allow to strictly regulate the load, widely use the necessary modes of muscle activity and program the nature of the effort manifestation, save time for the athlete $[6,7]$.

There are few literary sources devoted to training devices in throwing, percussion and universal martial arts. Further improvement of the training methodology largely depends on a rational combination of the physical qualities development processes and formation of motor skills in athletes. Therefore, one of the most effective ways is the use of various training devices in the athletes training. According to the definition, a simulator is a set of devices that allows to reproduce exercises and their main elements in specially created artificial conditions that allow to regulate the modes of performing movements and their appropriate changes [8-10].

The original classification of simulators is given by V. N. Platonov [11]. He suggests distinguishing six main groups. Two of them are directly used in the system of training high-class athletes:

\footnotetext{
* Corresponding author: yprof@yandex.ru
} 
1. Strength simulators with variable resistance for simultaneous manifestation of strength qualities and mobility in the joints. The design of them is based on the use of levers, eccentric disks (cams), blocks and a set of loads (stacks).

2. Simulators that allow to combine the process of developing motor skills with technical improvement.

Inertia-free Heyvus ${ }^{\circledR}$ base line simulators combine the characteristics of both mentioned groups. The first difference between these simulators and traditional power ones is that they use an elastic element as a loader. It ensures absolute safety of performing the exercise. The second difference lies in the special design of the loading mechanism - a complex of technical components consisting of an elliptical drum, a pendulum stabilizer and a lever. Exercises using any Heyvus ${ }^{\circledR}$ simulator are primarily aimed at timely voluntary relaxation of the tense muscle. It is directly involved in the performance of motor actions. In the elementary cycle of exercising, the muscle contracts in a concentric mode. After completion of contraction in this mode, an arbitrary switch to relaxation occurs, bypassing the eccentric mode, where the probability of destruction of muscle structures is high [12]. The return force of the elastic loader "forces" the muscle to gently stretch (lengthen). At the end of the cycle, the length of the muscle slightly exceeds its original length. There is a passive mechanical stimulation of the muscle. The value of this excess is due only to inertia in the growing human biokinematic chain. The muscle stimulation in the form of a long single or a series of short-time pull-ups causes gene expression. It follows that a relaxation exercise on the Heyvus ${ }^{\circledR}$ simulator can initiate the recovery of muscle structures directly during the exercise, and not outside of it $[12,14]$.

In this regard, we are interested in the processes of muscle relaxation in sports activities, the relaxation characteristics of skeletal muscles. Muscle relaxation manifests itself in changes in many physiological parameters, the rate of skeletal muscles relaxation. The rate of relaxation is no less important characteristic of the body's functional capabilities than the contractile characteristics of muscles [15-21]. This follows from the fact that any dynamic work is performed by a person by repeating cycles of movement with alternating periods of muscle contraction and relaxation. It requires optimal coordination and ordering of the tension levels of muscle groups. The importance of muscle relaxation is closely associated with the functional state of the Central nervous system (CNS). The muscle relaxation may be an underlying factor for improvement of most motor qualities necessary for highly effective training of highly skilled fighters on a belt.

\section{Materials and methods}

The problem of this study is the need of effective means development and physical training methods solving. The methods should include appropriate combinations of all training aspects with the definition of accurate quantitative relationships in accordance with the periods of the annual training cycle. At the same time, the main task of the search part of the study was to specify the features of the physical training tools. They are implemented in the training process of wrestlers in belts. To solve this problem, a pedagogical experiment was conducted within the framework of a one-year macrocycle. The only difference between the training methods of the experimental group of wrestlers was that they performed exercises on the "Runner" simulators from the Heyvus ${ }^{\circledR}$ baseline during the training session. The Heyvus ${ }^{\circledR}$ simulators were used in the following modes:

- twice a week for 45 minutes to develop speed, speed-strength qualities and strength endurance;

- daily for 30 minutes to improve the relaxation function of a large group of thigh muscles.

Selection of "Runner" simulators from the Heyvus ${ }^{\circledR}$ base line turned out to be not accidental - they allow to improve motor skills. They are the most important when performing most technical actions by athletes in belt wrestling.

The experimental method of working with wrestlers involved the use of inertia-free simulators in three modes:

1. Retractor (training in relaxation skills).

2. Relaxation (relaxation after a training session).

3. Developing (increasing the level of associated physical qualities).

There are several guidelines for training on inertia-free simulators:

- breaks between approaches should not exceed 60 seconds;

- in exercises aimed at developing general and special endurance, the pace of movements should be close to the pace of movements at a competitive distance;

- in exercises to develop strength (exercises with high and extreme resistance), the interval between approaches is $2-3$ minutes.

The loads dosage. When training aimed at developing general endurance, the duration of work on the inertia-free simulator is $20-25$ minutes - this is enough for deep muscle development.

The following series are recommended:

a) $5 \times 5$ minutes, rest interval- 60 seconds;

b) $10 \times 2 \mathrm{~min}$, rest interval-1-30 sec.

C) $3 \times(2 \times 2 \mathrm{~min})$ rest interval- $60 \mathrm{sec}$;

d) $20 \times 1 \mathrm{~min}$, rest interval-10 sec.

The heater resistance of the inertia-free simulator is selected so that the second half of the approach is performed with a certain overcoming of fatigue. The pace is moderate- $45-60$ movements per minute. The necessary increase in load is made by gradually increasing the resistance. 
When training aimed at increasing special endurance, the total duration of work on the inertia-free simulator is reduced to 15-20 minutes, but the intensity of the impact on the wrestler's body increases. The following series are recommended:

a) $8 \times 2$ min, rest interval-60 seconds, resistance $65-70 \%$ of the maximum, the pace in the first minute is moderate (45-50 movements per minute), in the next 30 seconds the pace increases and in the last 30 seconds it is brought to the maximum;

b) $16 \times 1 \mathrm{~min}$, rest interval-30 seconds, resistance $75-80 \%$ of the maximum, the rate continuously increasing to the maximum in the last 15 seconds;

C) $4 \times(2 \mathrm{~min}+1 \mathrm{~min}+1 \mathrm{~min})$, the rest interval between the series is 60 seconds, within the series-30-60 seconds. In the two-minute approach, the weight is $75 \%$ of the maximum, and the pace of movement increases to the maximum in the last 30 seconds. In the first minute approach, $1.5-2 \mathrm{~kg}$ is added to the resistance of the loader, the pace is average. In the second minute approach, $1.5-2 \mathrm{~kg}$ is added to the resistance and the athlete tries to maintain or even increase the pace shown in the previous minute approach.

To control the contractile and relaxation characteristics of skeletal muscles, the functional state of the central nervous system and the neuromuscular system, we used the method of computer polymyography developed by U. V. Vysochin [15].

The method of computer polymyography is based on synchronous graphic recording of the skeletal muscles' bioelectric activity -electromyograms and effort dynamograms developed by the muscle group in arbitrary tension and relaxation in the isometric mode.

\section{Results and discussion}

During the experiment, significant changes were found in the general and special physical fitness indicators of the wrestlers from experimental and control groups. But the wrestlers of the experimental group showed significantly better performance compared to the results of the control group.

Increasing the load in the exercises, you should follow the General rule: if the athlete successfully handles the load and keeps the pace, a further increase in loads should not go the way of increasing the pace but increasing the resistance.

When training to increase maximum strength, the athlete performs 6-8 sets of 4-10 movements each. The rest interval between approaches is up to 2-3 minutes. With each approach, the resistance should steadily increase. In the last 2-3 approaches, you can reduce the number of movements by 2-3, but the resistance should be maximum.

Planning a training session on simulators.

Exercises on non-inertia simulators can be included in the training during all periods of training.

When planning special work on inertia-free simulators, it is necessary to take into account the nature of the training loads provided for the class. It is advisable that these loads are unidirectional. This means that if you plan to work primarily at high speed, then you should also perform power or speed-power exercises on non-inertia simulators.

The first month of the preparatory period should be devoted mainly to general physical training. It includes a wide range of different exercises.

The first days of training should start with moderate loads, gradually preparing the system of muscles and ligaments for this type of exercise.

After being drawn in to work on inertia-free simulators from the Heyvus ${ }^{\circledR}$ base line, the loads should increase significantly.

Work on inertia-free simulators should last during the competitive period of wrestlers training. It is especially important at this time for those athletes who attach great importance to physical training.

In the days of the competition, work on inertia-free simulators runs in the cool down mode.

Thus, we can make the following summary:

- means of physical training in the training process of wrestlers on belts significantly determine the size of the increase in physical fitness indicators;

- traditional construction of wrestlers on belts training process with application of power exercises with weights and on simulators provides only moderate growth of physical fitness indicators and considerably lags behind modern tendencies of wrestling development;

- introduction of exercises based on inertia-free simulators of the Heyvus ${ }^{\circledR}$ base line as a priority means of physical training in the training process of belt wrestlers has a more significant positive impact on physical fitness indicators;

- the information obtained as a result of the experiment can be effectively implemented in the development of an experimental physical training program with an emphasis on the use of inertia-free simulators of the Heyvus ${ }^{\circledR}$ base line in the training process of belt wrestlers.

\section{Conclusions}

The analysis of the data on the ways to effectively combine physical training tools of various types and directions in the training process of wrestlers in belts allows us to draw the following conclusions: 
We will list the features, advantages and areas of the Heyvus ${ }^{\circledR}$ base line inertia-free simulators use:

1. Working on simulators in the muscle relaxation mode allows immediately after muscle tension proceed to full-fledged muscle relaxation. The muscle stretches to its physiological length, initiating an urgent restoration of its structure through the mechanism of gene expression. The residual tension that occurred in the muscle earlier (before the exercise) is eliminated. Thus, the athlete's neuromuscular system acquires a higher level of functional state.

2. The Heyvus ${ }^{\circledR}$ line of simulators makes it possible to form the correct motor skill according to the phases of motor action by appropriately localizing the maximum muscle tension.

3. Taking into account the safety of working on simulators for joints and muscle tendons, they can be successfully used in the training system for young athletes in order to form the correct motor skills from the first steps in sports. Thus, these simulators have the potential to solve well-known problems of early specialization in sports.

4. The advantages of the considered simulators over power ones guarantee their use as a mechanotherapy tool in the rehabilitation of athletes after injuries to joints, ligaments, muscles and their tendons.

5. The Heyvus ${ }^{\circledR}$ line of simulators can be used to develop the motor quality of strength. For this purpose, at the end of the concentric muscles contraction period, it is enough not to relax them, but to start a full-fledged eccentric contraction.

6. Compared to weight training devices that do not allow operation in the muscle relaxation mode, the Heyvus ${ }^{\circledR}$ base line simulators have a number of undeniable advantages. The main one is that two goals are achieved simultaneously by the same technical means:

- immediately after the power load, there is an urgent restoration of performance by including exercises based on inertia-free simulators in the muscle relaxation mode in the training session;

- the combined development of motor qualities with strength, endurance and speed qualities are provided, the relaxation characteristics of the muscles are improved.

\section{References}

1. V.S. Begidov, A.N. Parkhomenko, V.V. Shiyan, Theory and practice of physical culture, 11, 45-47 (1988)

2. S.I. Telyuk, Candidate's thesis (M., 1984)

3. A.S. Kuznetsov, Z.M. Kuznetsova, Russian Journal of Physical Education and Sport, 14(4), 5-7 (2019). DOI: 10.14526/20704798-2019-14-4-5-7

4. V.V. Shiyan, Means and methods of improving anaerobic capabilities of highly qualified judoists (Fon, M., 2001)

5. O.P. Yushkov, V.P. Serdyuk, S.M. Repnevsky, Sports wrestling: Yearbook (Physical Culture and Sport, M., 42-43, 1977)

6. V.S. Topchiyan, G.I. Popov, Modern aspects of planning the training of young athletes: theses of the VII all-Union scientific and practical conference (Tallinn, September 1981) (Tallinn, 1151-153, 981)

7. A.D. Skripko, Special training of athletes' sprinters (Higher school, Mn., 12-15, 1977)

8. .T.N. Makarov, E.N. Fedorov, Special performance and mechanisms of its development, 6, 79-85 (1979)

9. I.P. Ratov, I. P. Doctor's thesis (M., 1972)

10. R.A. Akmaletdinov, Historical aspects and innovations in the development of traditional and folk sports in the context of the World's people culture: collection of scientific articles of the International scientific and practical conference (RIC Bashifk, UFA, 6-21, 2007)

11. V.N. Platonov, Training of qualified athletes (Kiev, 1986)

12. R.A. Akmaletdinov, V.A. Demidov, E.V. Ostrovsky, F.A. Shemuratov, The need to motivate the population's interest in physical culture and sports, the formation of a healthy lifestyle: materials of the all-Russian scientific and practical Conference (RCIM, Kazan, 2, 101-102, 2004)

13. M.J. Alter, The science of flexibility (Olympic literature, Kiev, 2001)

14. A.J. McComas, Skeletal muscles (Olympic literature, Kiev, 2001)

15. U.V. Vysochin, Theory and practice of physical culture, 6, 26-29 (1978)

16. U.P. Denisenko, R.A. Gumerov, A.I. Morozov, A.Kh. Mardanov, Theory and practice of physical culture, 9, 69-72 (2018)

17. A.G. Kamchatnikov, N.N. Sentyabrev, M.M. Bogomolova, V.V. Chemov, M.V. Manzhela, M.U. Popov, Questions of balneology, physiotherapy and therapeutic physical culture, 96(2), 80-81 (2019)

18. I.V. Levshin, E.N. Kuryanovich, S.A. Trapeznikov, Theory and practice of physical culture, 8, 48-49 (2019)

19. N.N. Sentyabrev, Directed relaxation of the body during intense human muscle activity (VGAFK, Volgograd, 2004)

20. Yu.P. Denisenko, A.M. Akhmetov, L.G. Yatsenko, A.A. Geraskin, Yu.N. Ertman, International Scientific and Practical Conference on Education, Health and Human Wellbeing (ICEDER 2019), Advances in Social Science, Education and Humanities Research, 396, 428-431 (2019)

21. Yu.P. Denisenko, A.M. Akhmetov, D.B. Paramonova, N.N. Selivyorstova, First International Volga Region Conference on Economics, Humanities and Sports,/Advances in Economics, Business and Management Research, 114, 636-639 (2019) 